\title{
Comparisons of Nonlinear Estimators for Wastewater Treatment Plants
}

\author{
H. F. Wahab*, R. Katebi* and R.Villanova** \\ ${ }^{*}$ Industrial Control Centre, \\ *Department of Electrical and Electronic Engineering, University of Strathclyde, G1 1QE, Glasgow, UK. \\ Email: \{r.katebi, hamimi.abdulwahab\}@eee.strath.ac.uk \\ **Dept.de Telecomunicació i Enginyeria de Sistemes, ETSE, UAB, Email: Ramon.Vilanova@uab.cat
}

Keywords: Nonlinear state estimation, Wastewater Systems, Kalman filter, H-infinity filter, state-dependent filter, Unscented Kalman filter

\begin{abstract}
This paper deals with five existing nonlinear estimators (filters), which include Extended Kalman Filter (EKF), Extended H-infinity Filter (EHF), State Dependent Filter (SDF), State Dependent H-Infinity Filter (SDHF) and Unscented Kalman Filter (UKF) that are formulated and implemented to estimate unmeasured states of a typical biological wastewater system. The performance of these five estimators of different complexities, behaviour and advantages are demonstrated and compared via nonlinear simulations. This study shows promising application of UKF for monitoring and control of the process variables, which are not directly measurable.
\end{abstract}

\section{INTRODUCTION}

$\mathrm{T}^{\mathrm{h}}$ he design and application of state estimator in biological wastewater treatment plant (WWTP) has been an active area of research over the past decades. The difficulty of determining the states of a dynamic behavior of the system via few available measurements has led to the development and implementation of a wide variety of state estimation algorithms. Some application of the different estimation techniques to WWTP have been discussed in [1],[2],[3].

The most widely used suboptimal filter, the Extended Kalman Filter (EKF) which facilitates the Jacobian of the nonlinearity in the dynamics is used routinely and successfully in many practical applications including WWTP [4],[5]. Under weak nonlinearity, the EKF, have demonstrated precise performance, but diverge under more highly nonlinear cases and its estimates highly dependent on the accuracy of model used [6],[7].

Since EKF is biased and not robust to the modeling uncertainties [8], the extended H-infinity filter (EHF) has been an alternative to minimizes the worst possible effect of the modeling errors and additive noise on the signal estimation errors [9],[10]. There has been a number of different approaches to the formulation of the EHF in the literature, where different approaches all lead to extensively different equations [11]. Thus, making the entire field of $H_{\infty}$ filter rather difficult to implement and use. However, despite its difficulty and extra tuning required by $H_{\infty}$ filter, it is still worthwhile to consider this type of filter for its robustness [11],[12].

In contrast to the EKF and EHF which are based on Taylor series type linearization, another different approach to state estimation of nonlinear systems based on parameterization that brings the nonlinear system to a linear structure having state-dependent coefficients (SDC) is the State Dependent Riccati Filter (SDF) [13]. It is also shown in [14] that, the SDF parameterization is not unique and can create extra degrees of freedom that are not available in traditional filtering methods to avoid singularities and loss of observability.

EHF and SDF have their own respective advantages in terms of estimator robustness [15],[16] that can be manipulated to develop a new algorithm called State Dependent H-Infinity Filter (SDHF). This new filter employs a state- dependent model and H-infinity design technique to estimate the system states.

Another filter that can avoid the cumbersome evaluation of the Jacobian matrices and does not approximate the nonlinear models is the Unscented Kalman filter (UKF) [17],[18]. The UKF uses a parameterized set of sample points, called 'sigma point' according to a specific deterministic sampling to model the nonlinearity [17]. Using the true nonlinear models, UKF can give more accurate results than the linearization technique algorithm for propagating mean and covariance [19].

Although these algorithms have been widely studied in the literature, the performances and applications of the EHF, SDF, SDHF and UKF for the activated sludge process in WWTP have seldom been discussed. Therefore, the objective of this paper is to produce a comparative study of the well known EKF and the above mentioned filters for state estimation of a biological WWTP. The remainder of this paper is organized as follows: Section II presents a brief formulation of EKF, EHF, SDF, SDHF and UKF algorithms. Section III is dedicated to a brief description of biological process used. The comparison between the filters is performed by simulation studies in Section IV. A general conclusion ends the paper. 


\section{NONLINEAR FILTERS FORMULATION}

The algorithms of five different nonlinear filters are formulated in this section. The nonlinear system is assumed to be piecewise observable and controllable.

\section{A. Extended Kalman Filter (EKF)}

The EKF simply approximate nonlinear dynamics $f$, and output function $h$ with first order Taylor series expansion around the current estimate by discarding the second and higher order terms to evaluate covariance and the filter gains. Table I presents the summary of EKF algorithm.

TABLE I

EKF ALGORITHM

\begin{tabular}{|c|c|}
\hline \multirow{2}{*}{$\begin{array}{l}\text { System } \\
\text { model: }\end{array}$} & $\dot{x}(t)=f(x(t), u(t))+w(t) \quad ; w(t) \sim N(0, Q(t))$ \\
\hline & $y(t)=h(x(t))+v(t) \quad ; v(t) \sim N(0, R(t))$ \\
\hline $\begin{array}{l}\text { Initial } \\
\text { Conditions: }\end{array}$ & $\hat{x}\left(t_{0}\right)=E\left[x\left(t_{0}\right)\right] \quad ; P\left(t_{0}\right)=\operatorname{Var}\left[x\left(t_{0}\right)\right]$ \\
\hline Filter Gain: & $K(t)=P(t) H^{T}(t) R^{-1}(t)$ \\
\hline \multirow[t]{3}{*}{$\begin{array}{l}\text { Error } \\
\text { Covariance: }\end{array}$} & $\dot{P}(t)=A(t) P(t)+P(t) A^{T}(t)$ \\
\hline & $-P(t) H^{T}(t) R^{-1}(t) H(t) P(t)+Q(t)$ \\
\hline & where: $\left.A \equiv \frac{\partial f}{\partial x}\right|_{\hat{x}(t), u(t)},\left.\quad H \equiv \frac{\partial h}{\partial x}\right|_{\hat{x}(t)}$ \\
\hline $\begin{array}{l}\text { State } \\
\text { Estimator: }\end{array}$ & $\hat{\dot{x}}(t)=f(\hat{x}(t), u(t))+K(t)[y(t)-H(t) \hat{x}(t)]$ \\
\hline \multicolumn{2}{|c|}{$\begin{array}{l}\text { where } x(t) \in \mathfrak{R}^{n} \text { is the state vector and } y(t) \in \mathfrak{R}^{m} \text { is the measurement } \\
\text { vector. } w(t) \text { and } v(t) \text { represent the uncorrelated zero-mean Gaussian } \\
\text { process and measurement noise with covariance } Q(t) \text { and } R(t) \text {, } \\
\text { respectively. }\end{array}$} \\
\hline
\end{tabular}

\section{B. Extended H-Infinity Filter (EHF)}

As can be seen from Table II, the structure of EHF estimator is similar to EKF. The main difference is calculating the filter gain, where the inclusion of $\gamma$ term tends to increase the norm of $P$. This will in turn increase the gain, $K$ and hence make the estimator more responsive to the measurement compared to EKF [20]. The value of $\gamma$ is reduced in steps until one of the eigenvalues of $P$ becomes imaginary or negative. For the optimal solution $P$ should stay positive definite $(P>0)$. However, an alternative scheme introduced in [21] can be utilized using the time decreasing exponential function.
TABLE II

EHF ALGORITHM

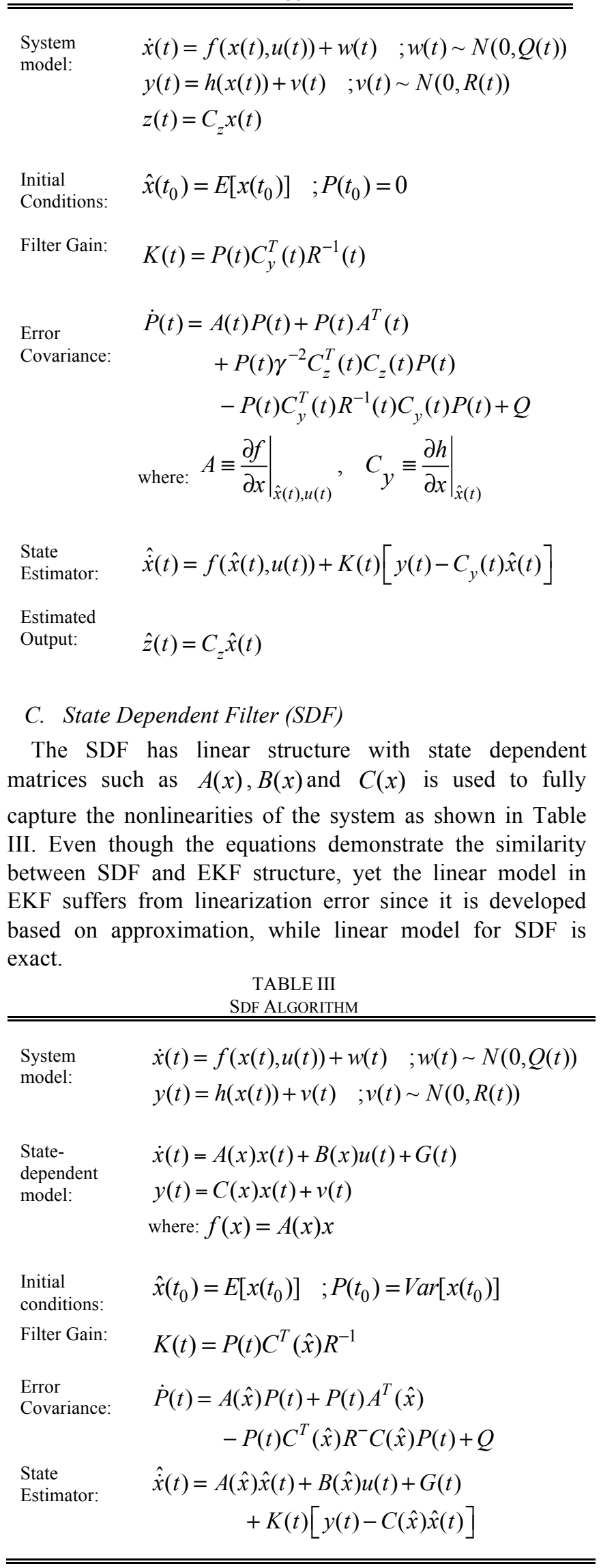




\section{State-Dependent H-Infinity Filter (SDHF)}

SDHF employs a state- dependent model and H-infinity design technique to estimate the system state. It is aimed at combining the advantages of both SDF and EHF. Table 1V shows the algorithm of this filter.

TABLE IV

SDHF ALGORITHM

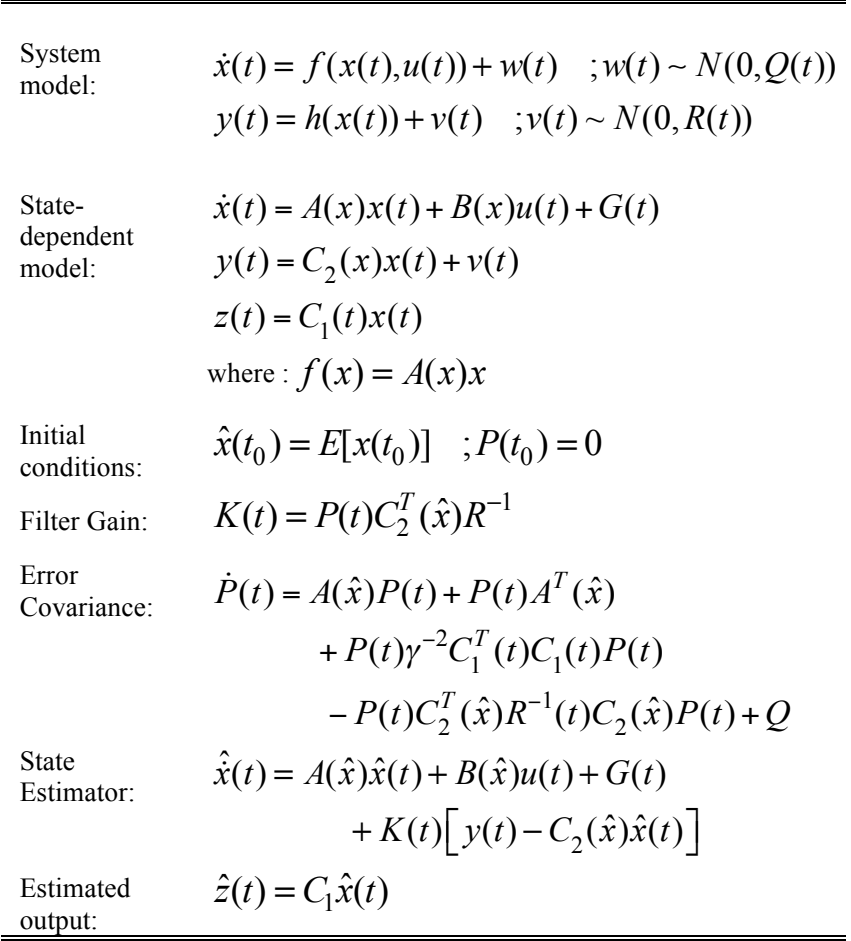

\section{E. Unscented Kalman Filter (UKF)}

The UKF does not approximate the nonlinear process and observation models; it uses the true nonlinear models and the Gaussian probability density by a number of deterministically chosen points, called sigma point. The UKF algorithm is based on the unscented transformations, which are more accurate than the linearization technique algorithm for propagating mean and covariance. The algorithm presented in Table $\mathrm{V}$ is the most general form of UKF.

\section{SimUlation STUDIES}

In this paper, the activated sludge process (ASP), which is the most generally applied biological wastewater treatment method, will be used for nonlinear state estimation study. The Activated Sludge Model No. 1 (ASM1) presented by the International Water Association (IWA) [22] are generally accepted as the reference model or benchmark model, which was primarily developed for municipal ASPs to describe the removal of organic carbon substances and nitrogen. Other models that improve and extend the capabilities of ASM1 are ASM2, ASM2d and ASM3 which can be found in [23].
Since the use of such models is complicated and for the early stage of estimator design, the biological process adopted in the present work is a simple model of an activated sludge process (ASP) proposed by Nejjari, Roux et al. [24]. The model truly respects the objectives of the process and was used widely in literature. It consists of an aeration tank and a secondary clarifier that is necessary for the settling of the biomass and its recycling as displayed in Fig.1.

TABLE V UKF ALGORITHM

$$
\begin{array}{ll}
\text { System model: } & x_{k+1}=f\left(x_{k}, u_{k}, t_{k}\right)+w_{k} ; w_{k}: N\left(0, Q_{k}\right) \\
& y_{k}=h\left(x_{k}, t_{k}\right)+v_{k} ; \quad v_{k}: N\left(0, R_{k}\right) \\
\text { Initial } & \hat{x}_{0}^{+}=E\left[x_{0}\right], \quad P_{0}=E\left[\left(x_{0}-\hat{x}_{0}^{+}\right)\left(x_{0}-\hat{x}_{0}^{+}\right)^{T}\right. \\
\text { Conditions: } & x_{k-1}^{(i)}=x_{k-1}^{+}+\tilde{x}^{(i)}, i=1, \ldots, 2 n \\
\text { Sigma point: } & \text { where: } \\
& \tilde{x}^{(i)}=\left(\sqrt{n P_{k-1}^{+}}\right)^{T}, i=1, \ldots, n \\
& \tilde{x}^{(n+i)}=-\left(\sqrt{n P_{k-1}^{+}}\right)_{i}^{T}, i=1, \ldots, n \\
\text { Time update } & x_{k}^{(i)}=f\left(x_{k-1}^{(i)}, u_{k-1}^{(i)}, t_{k}\right) \\
\begin{array}{ll}
\text { 2n sigma points } \\
\text { for nonlinear } \\
\text { process: }
\end{array} & x_{k}^{-}=\frac{1}{2 n} \sum_{i=1}^{2 n} x_{k}^{(i)} \\
\text { State vector: } & P_{k}^{-}=\frac{1}{2 n} \sum_{i=i}^{2 n}\left(x_{k}^{(i)}-x_{k}^{-}\right)\left(x_{k}^{(i)}-x_{k}^{-}\right)^{T}+Q_{k} \\
\text { Covariance: } &
\end{array}
$$

Measurement

update

2n sigma points for measurement:

Measurement:

$y_{k}^{(i)}=h\left(x_{k}^{(i)}, t_{k}\right)$ $y_{k}^{-}=\frac{1}{2 n} \sum_{i=1}^{2 n} y_{k}^{(i)}$

Covariance:

$P_{y}=\frac{1}{2 n} \sum_{i=i}^{2 n}\left(y_{k}^{(i)}-y_{k}^{-}\right)\left(y_{k}^{(i)}-y_{k}^{-}\right)^{T}+R_{k}$

Cross

covariance:

$P_{x y}=\frac{1}{2 n} \sum_{i=0}^{2 n}\left(x_{k}^{(i)}-x_{k}^{-}\right)\left(y_{k}^{(i)}-y_{k}^{-}\right)^{T}$

Filter Gain:

$$
K_{k}=P_{x z} P_{z}^{-1}
$$

Filter Estimates:

$x_{k}^{+}=x_{k}^{-}+K_{k}\left(y_{k}-y_{k}^{-}\right)$

Covariance:

$$
P_{k}^{+}=P_{k}^{-}-K_{k} P_{z} K_{k}^{T}
$$




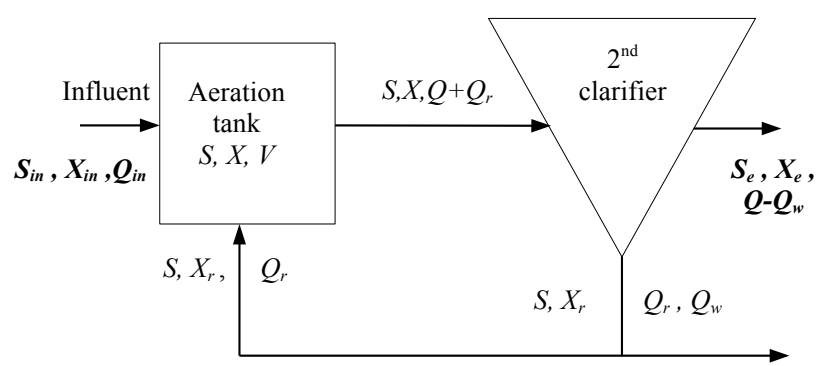

$S_{r}, X_{r}, Q_{w}$

Fig. 1. Activated sludge reactor for the filter application

In the aeration tank, the wastewater is aerated with oxygen including carbonaceous oxidation and nitrification where $Q$ represents the secondary influent flow rate; $Q_{r}$ the return sludge flow rate; $Q_{w}$ the waste activated sludge flow rate and $X_{e}$ the effluent suspended solids. The mass balance on the aerator and the settler are described by the set of nonlinear differential equations [24]:

$$
\begin{gathered}
\dot{X}(t)=\mu(t) X(t)-D(t)(1+r) X(t)+r D(t) X_{r}(t) \\
\dot{S}(t)=-\frac{\mu(t)}{Y} X(t)-D(t)(1+r) S(t)+D(t) S_{i n} \\
\begin{array}{r}
\dot{C}(t)=-\frac{K_{o} \mu(t)}{Y} X(t)-D(t)(1+r) C(t) \\
+K_{L a}\left(C_{S}-C(t)\right)+D(t) C_{i n}
\end{array} \\
\dot{X}_{r}(t)=D(t)(1+r) X(t)-D(t)(\beta+r) X_{r}(t)
\end{gathered}
$$

where $X(t), S(t), C(t)$ and $X_{r}(t)$ are the state variables representing the biomass, the substrate, dissolved oxygen and the recycled biomass concentrations, respectively. $D(t)$ is the dilution rate and the parameter $r\left(r=Q_{r} / Q\right)$ and $\beta$ $\left(\beta=Q_{w} / Q\right) . S_{\text {in }}$ and $C_{i n}$ corresponds to the substrate and dissolved oxygen concentrations in the feed stream, respectively. The kinetic of the cell mass production are defined in terms of the specific growth, $\left(\mu=r_{g} / X\right)$ and the yield of cell mass, $Y$; the constants $C_{S}$ and $K_{L a}$, represent the dissolved oxygen saturation concentration and the oxygen transfer rate coefficient $\left(K_{L a}=\alpha W\right)$ with $\alpha>0$ and $W=$ air flow rate), and the term $K_{o}$ is a switching constant. Biomass growth assumed a double Monod law in substrate and dissolved oxygen.

The kinetic model is given by [25]:

$$
\mu(t)=\mu_{\max } \frac{S(t)}{K_{s}+S(t)} * \frac{C(t)}{K_{c}+C(t)}
$$

\section{SIMULATION AND PERFORMANCE ANALYSIS}

A simulation study has been carried out to evaluate and compare the different estimation approaches applied to the ASP model. The simulation was performed with sampling time of $0.01 \mathrm{~s}$. The filters presented herein assumed constant parameter values in the nonlinear model. In some cases however, the parameters can evolve during process operation. The following estimation configuration was chosen: the biomass $X(t)$ and recycled biomass $X_{r}(t)$ are unavailable on-line and the estimation was carried out using the noisy measurements of substrate $S(t)$ and dissolved oxygen $C(t)$. The dilution rate $D(t)$ and the air flow rate $W(t)$ are the two control variables. The tuning procedure adopted for the filters are identical with the same process and measurement noise. The covariance matrices $P_{o}, Q$ and $R$ are assumed to be diagonal. The parameters and initial conditions used for simulation are given in Table VI.

TABLE VI

PARAMETERS AND INITIAL CONDITIONS

\begin{tabular}{ccc}
\hline \hline $\begin{array}{c}\text { Process } \\
\text { parameters }\end{array}$ & Kinetic parameters & Initial conditions \\
\hline$Y=0.65$ & $K_{s}=100 \mathrm{mg} / 1$ & $X(0)=217.78 \mathrm{mg} / 1$ \\
$r=0.6$ & $K_{c}=2 \mathrm{mg} / 1$ & $S(0)=41.28 \mathrm{mg} / 1$ \\
$\beta=0.2$ & $\mu_{\max }=0.15 \mathrm{~h}^{-1}$ & $C(0)=6.11 \mathrm{mg} / 1$ \\
$\alpha=0.018 \mathrm{~m}^{-3}$ & & $S(0)=435.58 \mathrm{mg} / 1$ \\
$K_{o}=0.5$ & & \\
$C_{s}=10 \mathrm{mg} / 1$ & \\
$C_{\text {in }}=0.5 \mathrm{mg} / 1$ & \\
$S_{\text {in }}=200 \mathrm{mg} / 1$ & \\
\hline \hline $\mathrm{m}=$ meter, $\mathrm{l}=$ liter, $\mathrm{h}=$ hour, $\mathrm{mg}=$ miligram
\end{tabular}

Under constant dilution rate $D(t)$ and the air flow rate $W(t)$, the estimation results for the unmeasured states, biomass $X(t)$ and recycled biomass $X_{r}(t)$ are displayed in Figs. 2-3 while the substrate $S(t)$ and dissolved oxygen $C(t)$ were not shown here. It shows that all the five filters have the ability to converge to its true states. Since the true states are difficult to observe because the filters provide almost the exact values, the quantitative analysis are presented. Three aspects are compared for filters: accuracy of state of each algorithm in terms of standard deviation of estimation error, Root Mean Squared Errors (RMSEs) with respect to its estimates and computation time.

Even though, there is no approximation involved in SDF and SDHF, in this study it is observed that the performance of the UKF is superior to the other filters as demonstrated by the standard deviation data in Table VII when using smaller value of process and measurement noise. The absolute estimation error for biomass $X(t)$ and recycled biomass $X_{r}(t)$ in Fig. 4-5 has confirmed the superiority of UKF compared to the other filters. UKF uses the unscented transformation to directly approximate the nonlinear system.

In this study, when both process and measurement noise are increased, SDHF and UKF demonstrate comparable performance, as shown in Table VII (Case B). The 
corresponding root mean square error (RMSE) for all the five filters, displayed in Fig. 6 also confirms this finding.

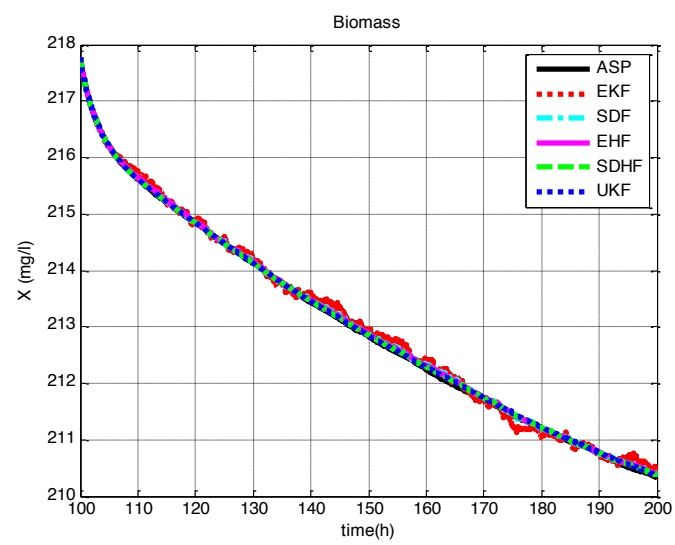

Fig. 2. True nonlinear states $X$ and its estimate

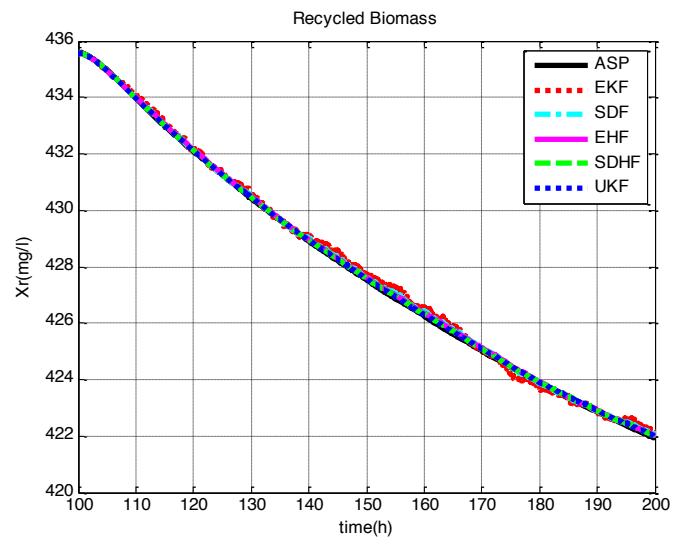

Fig. 3. True nonlinear states $X_{r}$ and its estimate

The most common case in the WWTP field is the one in which plant uncertainty is present in initial conditions [2]. Thus, in this study different initial conditions were given to the filters to observe the convergence properties for all the tested filters. As displayed in Fig. 7-8, the UKF converges more quickly than the other filters. It is demonstrated that initial state covariance have a significant impact on the UKF performance [26].

The computation time required for each method is display in Table VIII using Case A where simulations are performed in Matlab R2010b with a clock speed of $3.2 \mathrm{GHz}$ Pentium computer running Windows 7 using Matlab's built in function cpu time. It is observed that the SDF is significantly faster than EKF and other approaches. This is due to the fact the nonlinear model is transformed to a linear time varying model off-line and hence did not require much computational time as compared to the calculation of the Jacobian matrix in EKF. On the other hand, it is noted that the UKF and EKF have comparable computation times. Meanwhile, the extra tuning parameter gamma $(\gamma)$ in the formulation of EHF and SDHF which need to be iterated to find the best value of the gain has increased the CPU time for these filters.

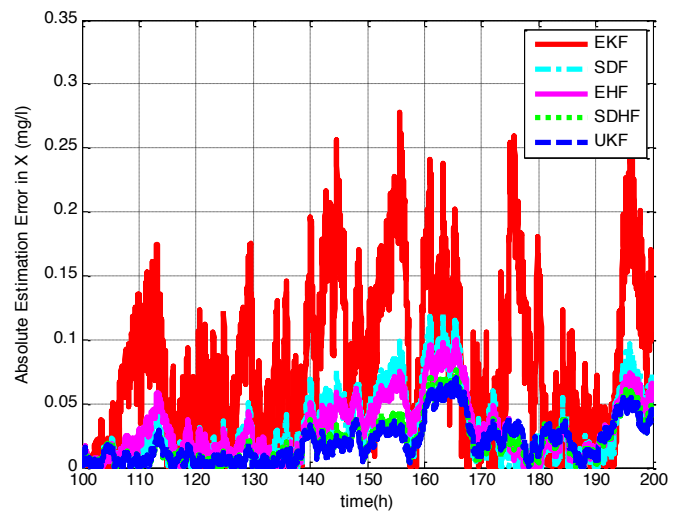

Fig. 4. Absolute estimation error in Biomass, $X$ (Case A)

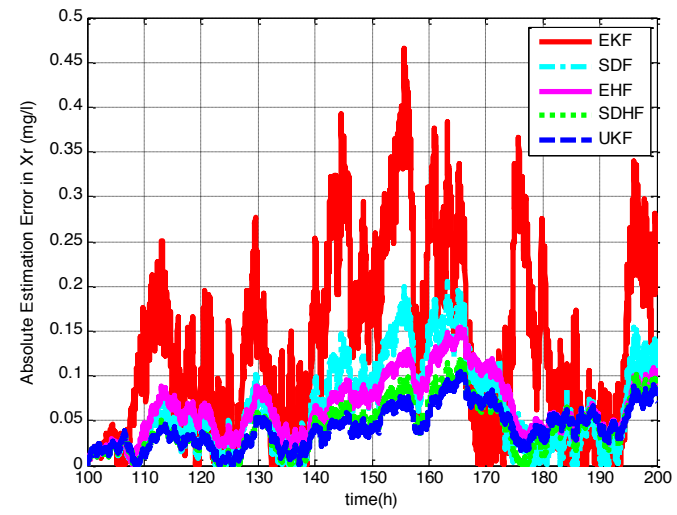

Fig. 5. Absolute estimation error in Recycled Biomass, $X_{r}$ (Case A)

TABLE VII

STANDARD DEVIATION OF ESTIMATION ERRORS

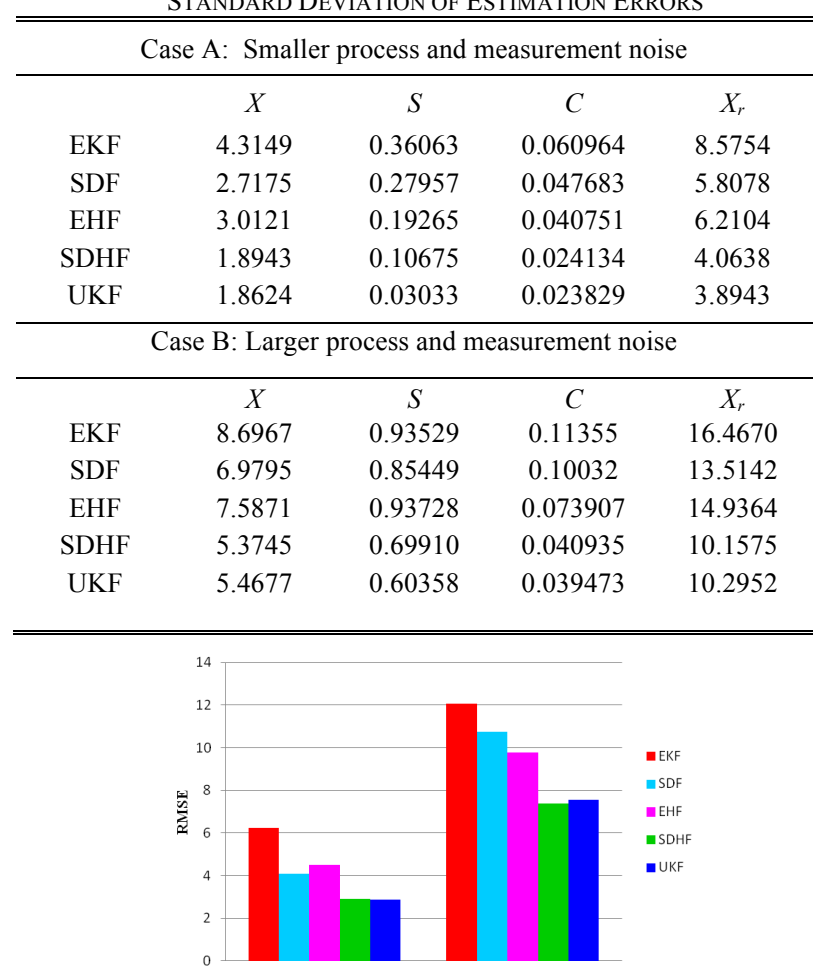

Fig. 6. Comparisons of RMSE for all filters 


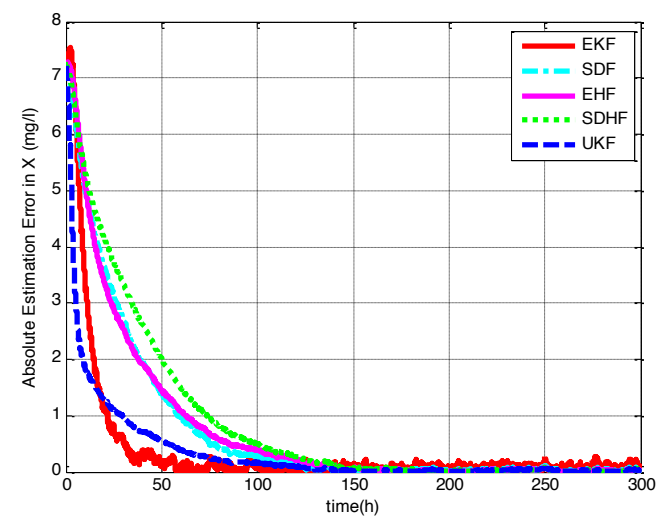

Fig. 7. Absolute estimation error in Recycled Biomass, $X_{r}$ with different initial condition

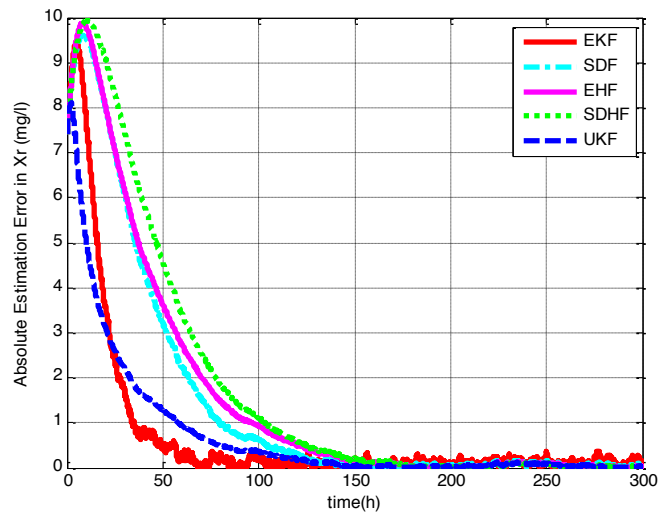

Fig. 8. Absolute estimation error in Biomass, $X$ with different initial condition

TABLE VIII

COMPUTATION TIME (SECONDS)

\begin{tabular}{ccccc}
\hline \hline EKF & SDF & EHF & SDHF & UKF \\
\hline 9.18996 & 8.7477 & 27.10380 & 19.02962 & 9.20124 \\
\hline \hline
\end{tabular}

\section{CONCLUSION}

In this paper the use of a number of estimation approaches to estimate unmeasured states of the activated sludge model proposed by Nejjari, Roux et al. [24] have been investigated and compared. Certainly, other techniques that are relevant to the field of WWTPs exist and the authors did not pretend to be exhaustive; but these approaches were chosen because of its conceptual simplicity and generality. A good compromise between the quality of the estimation and the difficulty of implementation should be taken into account when to choose or design a filter for a specific application. A satisfactorily tested filter in one application does not necessarily produce satisfactory result in other application.

To conclude, it is observed that all of the filters presented good convergence properties for WWTPs. The study shows UKF have better estimation accuracy and can be a costeffective preference to physical sensors for state estimation; thus, promising extended application of UKF for monitoring the process variables, which are not directly measurable in the treatment of waste water plant.

\section{ACKNOWLEDGMENT}

The first author is grateful to the Malaysian Ministry of Higher Education for financial support to carry out this research.

\section{REFERENCES}

[1]D. Dochain and P. Vanrolleghem, Dynamical modelling and estimation in wastewater treatment processes: Intl Water Assn, 2001.

[2]V. Alcaraz-González, et al., "Software sensors for highly uncertain WWTPs: a new approach based on interval observers," Water Research, vol. 36, pp. 2515-2524, 2002

[3]K. J. Keesman, "State and parameter estimation in biotechnical batch reactors," Control Engineering Practice, vol. 10, pp. 219-225, 2002.

[4]L. J. S. Lukasse, et al., "A recursively identified model for short-term predictions of NH4/NO3 - concentrations in alternating activated sludge processes," Journal of Process Control, vol. 9, pp. 87-100, 1999.

[5]C. F. Lindberg, "Control and estimation strategies applied to the activated sludge process," PhD, Uppsala University, 1997.

[6] A. Gelb, Applied optimal estimation: MIT press, 1974.

[7]I. Hoteit, et al., "A new approximate solution of the optimal nonlinear filter for data assimilation in meteorology and oceanography," Monthly Weather Review, vol. 136, pp. 317-334, 2008.

[8]S. Xu and P. Van Dooren, "Robust H filtering for a class of non-linear systems with state delay and parameter uncertainty," International Journal of Control, vol. 75, pp. 766-774, 2002.

[9]M. Grimble, "Optimal H robustness and the relationship to LQG design problems," International Journal of Control, vol. 43, pp. 351-372, 1986.

[10] M. Katebi and M. Grimble, "Design of dynamic ship positioning using extended H filtering," 1998, pp. 202-207.

[11] D. Simon, "From Here to Infinity-H (infinity) filters can be used to estimate system states that cannot be observed directly. In this, they are like Kalman filters. However, only H (infinity) filters are robust," Embedded Systems Programming, vol. 14, pp. 20-34, 2001.

[12] L. Xi-Mei, et al., "Fault diagnosis of High Voltage Direct Current system based on $\mathrm{H}_{\infty}$ filter," in IEEE International Conference on Robotics and Biomimetics, 2007, pp. 162-166.

[13] C. P. Mracek, et al., "A new technique for nonlinear estimation," in IEEE International Conference on Control Applications, 1996, pp. 338-343. [14] J. R. Cloutier, et al., "Nonlinear regulation and nonlinear H-infinity control via the state-dependent Riccati equation technique. I- Theory," 1996, pp. 117-130.

[15] F.Benazzi, "Software Sensor Designs for Urban Wastewater Systems," PhD, Department of Electronic and Electrical Engineering, University of Strathclyde, 2006.

[16] A. Iratni, et al., "On estimation of unknown state variables in wastewater systems," in IEEE Conference on Emerging Technologies \& Factory Automation 2009, pp. 1-6.

[17] E. A. Wan and R. Van Der Merwe, "The unscented Kalman filter for nonlinear estimation," in Adaptive Systems for Signal Processing, Communications, and Control Symposium, 2000, pp. 153-158.

[18] S. J. Julier and J. K. Uhlmann, "A new extension of the Kalman filter to nonlinear systems," 1997, p. 26.

[19] S. J. Julier, et al., "A new approach for filtering nonlinear systems," 1995, pp. 1628-1632 vol. 3.

[20] D. Simon, Optimal state estimation: Kalman, $H_{\infty}$ and nonlinear approaches: John Wiley and Sons, 2006.

[21] M. R. Katebi and M. J. Grimble, "Extended H-infinity filtering for dynamic ship positioning," in IFAC Conf. ACASP, Glasgow, UK, 1998.

[22] M. Henze, et al., "A general model for single-sludge wastewater treatment systems," Water Research, vol. 21, pp. 505-515, 1987.

[23] M. Henze, Activated sludge models ASM1, ASM2, ASM2d and ASM3 vol. 9: Intl Water Assn, 2000.

[24] F. Nejjari, et al., "Estimation and optimal control design of a biological wastewater treatment process," Mathematics and computers in simulation, vol. 48, pp. 269-280, 1999.

[25] G. Olsson, "State of the art in sewage treatment plant control," 1976.

[26] B. G. Saulson and K. C. Chang, "Nonlinear estimation comparison for ballistic missile tracking," Optical engineering, vol. 43, p. 1424, 2004. 